\title{
LOS PROCESOS DE TOMA DE DECISIONES EN EL SECTOR SANITARIO $Y$ SUS IMPLICACIONES POLITICAS
}

\author{
Antonio Ugalde \\ (Universidad de Texas)
}

\begin{abstract}
El creciente número de fracasos en la implementación de planes de sanidad en muchos países del mundo ha llevado a los gobiernos a examinar el proceso de toma de decisiones. En el presente trabajo se esbozan algunas dimensiones de un marco teótico que sirve para empezar a acumular información sobre las decisiones de salud. Se establecen cuatro grupos decisores: los directores o élites, programadores e implementadores, los consumidores de los servicios y los grupos de presión o de influencia. Cada uno de estos grupos toma decisiones que afectan los programas sanitarios desde que se inician hasta que se implementan o se paralizan. Es necesario estudiar las relaciones entre los decisores políticos, los programadores, los implementadores y los consumidores, así como los factores socio-culturales y políticos que influyen sus decisiones. La coordinación entre los diversos decisores que actúan en diferentes agencias sanitarias o relacionadas con la salud es otro aspecto importante si se quiere comprender los fallos en la implementación. Se conceptualizan dos tipos de coordinación, uno hotizontal y otro vertical, que se dan dentro de las agencias y entre las agencias sanitarias. Finalmente se señala cómo los fracasos en la implementación de programas afectan el proceso decisorio en sí mismo.
\end{abstract}




$$
\text { . }
$$


Durante los quince últimos años la preparación de planes de desarrollo económico se ha convertido en rutina administrativa en casi todos los países del mundo, y dentro de estos planes se han preparado los del sector sanitario. A medida que han ido pasando los años se ha llegado a la conclusión de que los planes sanitarios por sí solos, y lo mismo se puede decir de los planes de otros sectores, no son garantía de su ejecución. En ocasiones los planes han quedado archivados en las estanterías de los Ministerios de Sanidad, en otras muchas más la implementación no se ha ejecutado de acuerdo a los programas establecidos. ${ }^{1}$ En algunos círculos se ha llegado a considerar que los esfuerzos que se han hecho en aprender y en aplicar las técnicas de planificación no compensan los resultados obtenidos.

Algunas agencias internacionales de desarrollo han empezado a vislumbrar la necesidad de estudiar las causas de los fracasos en la implementación como parte del esfuerzo para mejorar la planificación sanitaria. Los fallos en la implementación implican que las políticas sanitarias y los programas derivados de esas políticas no están adecuados a la capacidad administrativa del sistema. ${ }^{2}$ Las organizaciones internacionales han empezado a interesarse por comprender la manera en que se preparan los planes sanitarios y la forma en que se determinan los programas. En otras palabras, se han empezado a interesar en la forma de cómo

1. Véase la obra de Arnaldo Gabalón, Una política sanitaria (Caracas: Publicaciones del Ministerio de Sanidad y Asistencia Social, 1965). Empieza a reconocerse que los factores políticos son una de las causas más importantes y menos estudiadas de los fallos en la implementación; Albert Waterston, Development Planning: Lessons of Experience (Baltimore: Johns Hopkins Press, 1965), p. 3.

2. Con esto no se quiere decir que con mejorar la administración pública se resuelven los problemas de implementación. Los planes y programas pueden estar tan en desacuerdo con la realidad política, económica y social que sean irrealizables. Los obstáculos a la implementación provienen con frecuencia de causas externas a la administración pública. Es interesante la discusión de Basil J. F. Mott, «Politics and International Planning», Social Science and Medicine, 8 (1974): 271-274. 
se toman decisiones en el sector sanitario o lo que pudiéramos llamar el proceso decisorio. Así, en 1970 la Organización Mundial de la Salud (OMS) de las Naciones Unidas patrocinó el primer estudio sobre el proceso decisorio en Colombia a invitación del entonces ministro de Sanidad doctor Antonio Ordóñez Plaja. En 1972 se repitió el experimento en Irán aunque en este país el proyecto fue de mucha menor envergadura. ${ }^{3}$ Estos estudios resultaron lo suficientemente satisfactorios como para que la OMS incorporarse en forma permanente el análisis de decisiones dentro del marco conceptual de las investigaciones de planificación sanitaria que se están llevando a cabo en Calí (Colombia) que es quizás uno de los esfuerzos más importantes en el Tercer Mundo. Casi paralelamente a estas actividades, en 1974, la Agencia Internacional para el Desarrollo (AID) del Departamento de Estado norteamericano reunió en Washington un pequeño grupo de expertos en planificación sanitaria para iniciar la preparación de un esquema teórico que sirviera de norma para estudiar los problemas de implementación de los planes sanitarios en aquellos países en vías de desarrollo en donde la agencia norteamericana financia proyectos sanitarios. En ese mismo año, el AID incorporaba en el estudio del sector sanitario de la República Dominicana el análisis de los procesos decisorios y también lo incluía en su anteproyecto de investigación del sector sanitario de Panamá. Estos comienzos por parte de las agencias internacionales no son más que modestísimos esfuerzos que requerirán muchos años/hombres más de investigación antes de que se empieze a desarrollar una teoría adecuada para comprender el proceso decisorio y así explicar los fallos en la implementación.

\section{Marco conceptual}

En otra publicación se ha presentado un modelo para estudiar burocracias públicas basado en el proceso decisorio que se puede resumir

3. Véase mi informe «A Study of Decision Making in the Health Sector of Iran», United Nations, World Health Organization, October, 1972. Se está preparando una publicación con los resultados del estudio en Colombia. Una pequeña sección fue publicada por Antonio Ugalde, «El proceso de toma de decisiones en el sector salud», Escuela Nacional de Salud Pública (Medellín: Universidad de Antioquía, 1972).

4. El proyecto de la República Dominicana ha sido ya terminado pero los resultados todavía no han sido publicados. Para el anteproyecto de Panamá, que probablemente no se llevará a cabo, véase Antonio Ugalde, «Planning for the Health Sector Assessment in Panama», American Public Health Association (Washington: mimeo, 1974). 
brevemente de la siguiente forma. ${ }^{5}$ Toda organización existe para la consecución de unos objetivos. Los objetivos así como los medios para alcanzarlos se definen a través de decisiones. Generalmente, para la consecución de los objetivos son necesarias varias decisiones que las hacen varios actores en todos los niveles administrativos. Las decisiones individuales, aunque son importantes como parte de un todo, no explican por sí solas los fallos en la implementación y por tanto es necesario introducir el concepto «serie de decisiones». La serie de decisiones es el conjunto de decisiones que se toman en el proceso de llevar a cabo un objetivo y en nuestro caso un programa sanitario. Pensamos que la unidad de análisis debe ser la serie de decisiones que empieza con una decisión inicial que pone en movimiento el proceso decisorio. Por ejemplo, la decisión de iniciar un programa de fluoración de aguas, tomada por el director general de sanidad, llevará a otras personas a decidir cuáles son los acueductos que se incluyen en el programa, qué tipo de equipos se van a comprar, cuántos operarios se van a entrenar para la administración de las sales, etc. Las decisiones iniciales son decisiones generales de alta política que no determinan la forma o alternativas de cómo se consigue el objetivo.

Las decisiones que siguen a la decisión inicial se dividen analíticamente en decisiones de programación y de implementación. Esta distinción es importante porque los actores y factores influyentes en el desarrollo de los programas son diferentes en las dos fases. Un ejemplo puede aclarar estos conceptos. Supongamos que se ha tomado una decisión inicial de aumentar los servicios sanitarios en las áreas rurales. De las varias alternativas posibles se escoge la organización de un servicio de medicina rural. Dependiendo del sistema político de cada país, estas decisiones se toman en el congreso, en el consejo de ministros, en juntas militares, en las Cortes, en comités o en la administración pública (Ministerio de Sanidad, seguridad social). Una vez que se determina la conveniencia de un programa de medicina rural empiezan las decisiones de programación que determinan qué se entiende por zona rural, quiénes son los médicos que deben servir, el tiempo del servicio, la remuneración, supervisión, etc. Una vez que el programa ha sido aprobado por la autoridad correspondiente (que será muy diferente en cada país y para cada programa) empieza la implementación. Durante la fase de implementación se toman también muchas decisiones que pueden llegar a modificar sustancialmente, e incluso paralizar, el programa.

5. Antonio Ugalde, «A Decision Model for the Study of Public Bureaucracies», Policy Sciences, 4 (1973): 75-84. 
Por ejemplo, los directores de los hospitales desde donde los médicos sirven a la población rural deciden que algunos días de la semana los médicos rurales deben atender la consulta externa del hospital en vez de visitar los puestos de salud rural. En otras ocasiones los vehículos de transporte se estropean, quedando los médicos rurales sin acceso a las zonas rurales, y el taller de reparaciones que depende del Ministerio de Obras Públicas, decide que la reparación de esos vehículos debe esperar varios meses puesto que a su juicio las pocas piezas de repuesto que tienen se deben utilizar para otros vehículos más importantes, y el Ministerio de Comercio Exterior no ha permitido más que la importación de un número determinado de accesorios. En definitiva el servicio médico no llega al campo como se había pretendido en la decisión inicial. Nótese que en caso de la decisión del transporte la decisión se ha tomado fuera del sector sanitario. En nuestro estudio detallado sobre decisiones en el Ministerio de Sanidad Pública de Colombia encontramos que los actores en el proceso de implementación no eran los mismos que los que habían participado en las decisiones de programación, y que a veces ni siquiera pertenecían al sector sanitario. Como se verá, uno de los problemas más difíciles es el problema de coordinación de decisiones entre las unidades del sector sanitario y las de otros sectores. Pensamos, pues, que la unidad de análisis es la serie de decisiones que van desde la inicial hasta la última que se toma cuando se termina de implementar o paralizar el programa.

Enfocando el estudio del sector sanitario desde el marco conceptual de decisiones se consigue un análisis dinámico que descubre los fallos en la implementación y sugiere formulas para intentar eliminarlos. En el presente trabajo nos limitaremos a exponer los aspectos siguientes del proceso decisorio: (1) definición de política sanitaria, (2) los tipos de decisiones y grupos decisores y (3) la coordinación de decisiones. Muchos otros aspectos importantes quedan fuera, como serían el estudio de la fase de programación, de la fase de implementación y la relación entre las dos (el uso de estadísticas, el significado de indicadores de salud para saber si el objetivo se ha conseguido o no).

\section{De políticas a programas}

Aunque el término política sanitaria es de uso común en todos los países del mundo, es difícil establecer cuál es la política sanitaria en cada país. El problema se origina en parte por la retórica que se usa en la política y por el fraccionamiento y dispersión de responsabilidades 
en materias de salud en tantas instituciones y agencias de gobierno. E1 Ministerio de Sanidad define parte de la política sanitaria, otra parte la puede definir el Ministerio de Educación en lo que se refiere a recursos humanos, o el Ministerio de Obras Públicas en lo que se refiere a sanidad ambiental. La falta de una definición operacional de salud aceptada por los gobiernos es otra razón por la que resulta difícil establecer en cada país cuál es su política sanitaria. No pretendemos llegar a definir lo que es política sanitaria aunque para efectos de la presente discusión la consideramos como el conjunto de objetivos generales establecidos por las personas o grupos con poder para tomarlos y que guían a los programadores en la selección de acciones sanitarias. La identificación de estas políticas tiene un interés más que académico, ya que toda evaluación del sector requiere conocer si las políticas se han cumplido, y por lo tanto hay que saber antes cuáles son. Ejemplos de objetivos o directivas generales son: la integración del seguro social y los servicios públicos de atención médica (Panamá), regionalización de los servicios sanitarios (Colombia), «privatización» de la atención médica (Irán), etc. Con frecuencia se encuentra que algunos de los objetivos generales son inconsistentes entre sí, y otras veces contradictorios. Por ejemplo, en Malasia existe una directiva que da preferencia al desarrollo rural del país y otra directiva que exige la centralización administrativa cuyo resultado ha sido la concentración de servicios sanitarios en las zonas urbanas. En Colombia se establecieron la descentralización administrativa y al mismo tiempo programas verticales para materno-infantil y cáncer. En Irán se introdujo la planificación en salud mientras que por razones políticas se establecían unidades autónomas de administración en salud sin coordinación (ejército, ministerio, cruz roja).

Las directivas generales de salud se encuentran esparcidas en documentos diversos: escritos y discursos de los presidentes nacionales, de los ministros de Sanidad, directores generales de seguridad social, directores de planificación nacional. También se pueden identificar en los planes nacionales de sanidad, en las actas oficiales de los congresos y en algunos países en leyes y ordenamientos políticos, códigos sanitarios. Nuestros estudios en varios países nos hace pensar que muy pocos tienen una política sanitaria claramente definida y consistente. Se puede añadir que el estudio de políticas es de gran utilidad para comprender las razones de las faltas de consistencia que muchas veces producen dificultades en la implementación de programas, y también para ayudar a la formulación de directivas más claras y precisas en el futuro.

6. Antonio Ugalde, «A Study of Decision...», op. cit. 
Conviene indicar que en países en que existen divisiones político-administrativas autónomas o semiautónomas (reinos, Estados, departamentos, provincias) los diversos niveles político-administrativos pueden tener políticas sanitarias independientes que también pueden ser contradictorias a las nacionales llegando incluso a crear ciertos conflictos constitucionales.'

El análisis de contenido es una de las técnicas más apropiadas para la identificación de las políticas sanitarias. ${ }^{8}$ Un método práctico de cotejar si las políticas sanitarias establecidas son algo más que retórica es a través del estudio de los presupuestos sanitarios. No podemos detenernos aquí a detallar las múltiples dificultades que existen en la mayoría de los países en el análisis de presupuestos (basta pensar, por ejemplo, en la diferencia entre lo presupuestado y lo ejecutado y los diferentes sistemas de contabilidad, en los presupuestos extraordinarios y a veces secretos) pero a pesar de las limitaciones los presupuestos son útiles para saber si ha habido cambios en la política sanitaria. El análisis de presupuestos es también una gran ayuda para establecer los poderes decisorios de personas y grupos. Si se mira desde el momento en que la unidad sanitaria más pequeña presenta su anteproyecto de presupuesto o gasto ejecutado al final del año se puede obtener una información valiosa sobre el poder de las personas que intervinieron a lo largo del año aumentando o recortando la petición inicial. Por ejemplo, en Colombia encontramos que el presupuesto se inicia en una unidad, de allí pasa a la división, al comité técnico, a la unidad de planificación del Ministerio, al ministro, a planificación nacional, al consejo de ministros, a presupuesto nacional, al ministro de Hacienda, al presidente, al congreso y a la tesorería. A cada paso se pueden dar modificaciones que aumentan fondos para algunos proyectos y los recortan para otros. A veces la tesorería cuando está corta de fondos decide qué programa debe esperar para recibir los desembolsos mensuales. Los retrasos en los desembolsos pueden producir efectos catastróficos en los programas sanitarios: una campaña de vacunación masiva de la triple se paralizó en un país cuando los vacunadores que habían sido especialmente entrenados para la campaña de-

7. Esta es una situación común en los Estados Unidos entre los Estados y el gobierno federal, entre el congreso y el ejecutivo. En Colombia el proceso de integración de servicios de sanidad fue paralizado por un juicio en la corte suprema que presentó el gobernador del departamento del Valle en contra del Ministerio de Sanidad.

8. Para una explicación de la técnica véase Ole R. Holsti, Content Analysis for the Social Sciences and Humanities (Menlo Park, Calif.: Addison Wesley, 1969). 
cidieron regresar a sus casas al no recibir su salario semanal, y así se echó a perder un programa bien diseñado de acuerdo a las técnicas más modernas.

En todo sistema político hay roles políticos y técnicos, y generalmente se encuentran los puntos de contacto entre los dos en la burocracia. Los ocupantes de las posiciones de contacto (linkage) facilitan la conversión de políticas en programas al servir como instrumentos de comunicación entre los dos grupos. La posición de contacto es crítica puesto que controla el flujo de comunicación; por una parte transmite la opinión de los técnicos y expertos a los políticos de forma que éstos puedan comprender las ramificaciones de sus decisiones y las alternativas que existen, y de otra parte, comunica a los técnicos las limitaciones politicas dentro de las cuales deben operar. El proceso de información es generalmente informal y por tanto el intermediario goza de laxitud para interpretar a su modo la información que recibe, para poner énfasis en ciertos aspectos que a su juicio son más importantes, incluso para descartar alternativas presentadas por los técnicos o añadir limitaciones a aquellas impuestas por los políticos. Por lo tanto, las decisiones que toma pueden ser tan o incluso más críticas que las de los dos grupos. A la persona que ocupaba una posición de contacto le preguntamos si se consideraba técnico o político, puesto que en realidad participaba en los dos mundos. Su respuesta fue muy expresiva cuando dijo: «Soy un politécnico», y sugiere la claridad con que los ocupantes de estos puestos perciben su rol. Generalmente son profesionales de la salud que tienen más permanencia en la burocracia que los políticos. Es necesario identificar las personas que ocupan las posiciones contacto para poder analizar sus decisiones; a veces ocupan los cargos que lógicamente debieran de acuerdo con el organigrama de la institución pero éste no es siempre el caso.

Nuestra discusión sobre posiciones que controlan el flujo de la comunicación entre políticos y técnicos nos lleva a considerar otras posiciones de contacto en la burocracia que son también de gran importancia en el proceso decisorio como son los puntos de contacto entre los programadores de salud y los distribuidores de los recursos (Ministerio de $\mathrm{Ha}$ cienda, tesorería nacional, planificación). Las personas que ocupan estas posiciones tienen igualmente una gran discreción para favorecer la canalización de recursos en una dirección o en otra y por tanto sus decisiones son también importantes. Igualmente, los puntos de contacto entre los niveles centrales y regionales son de importancia al canalizar la demanda de las regiones y procesar las comunicaciones del centro a la periferia. El estudio de las posiciones de contacto (linkages) merece 
mucha más atención de la que se le ha dado si es que queremos llegar a conocer por qué se toman ciertas decisiones y en definitiva por qué unas se implementan y otras no.

\section{Grupos decisores}

Los estudios de decisores generalmente se han limitado a estudiar las élites políticas (decision makers y/o influentials) y los grupos de presión porque su interés era determinar cómo se tomaban las decisiones que nosotros hemos llamado iniciales o de alta política." Probablemente se suponía que una vez tomadas las decisiones inidiales e importantes la ejecución estaba garantizada. Pero éste no es siempre el caso ni siquiera en los países industrializados que gozan de continuidad y «estabilidad» política y mucho menos en los países menos desarrollados. Hace unos veinte años se legisló la integración racial de las escuelas en los Estados Unidos y todavía está muy lejos de conseguirse. Nuestro marco conceptual nos lleva a analizar no solamente las decisiones iniciales sino también muchas otras que se toman en la programación e implementación, y por consiguiente al estudio de los autores de esas decisiones. Analíticamente se pueden identificar cuatro grupos o niveles de decisores: (1) el grupo que pudiéramos llamar directores, (2) el de ejecutivos, (3) el grupo de consumidores y (4) los grupos de influencia o de presión. ${ }^{10}$

Llamamos directores o élites a las personas que definen la política sanitaria. Las posiciones que ocupan las personas de este grupo varían de acuerdo con la naturaleza del sistema político. En donde existe una separación de poderes entre las ramas legislativa y administrativa (por ejemplo, los Estados Unidos, Dinamarca) los directores se encuentran en los dos y a veces se enfrentan en una lucha de poder decisorio. Los presidentes nacionales tienen el poder de vetar los proyectos de ley y paralizar las decisiones aprobadas por la ley a través de restricciones fiscales (impounding), y naturalmente pueden también solapadamente encontrar interpretaciones a las leyes para llevar a cabo acciones no pre-

9. Quizás uno de los estudios más conocidos es el de H. Eckstein, Pressure Group Politics: The Case of the Britisb Medical Association (Stanford, Calif.: Stanford University Press, 1960). Casi todos los estudios se han llevado a cabo en países de habla inglesa.

10. Los términos que hemos escogido son arbitrarios y no pretendemos ques sean los más correctos. En vez de directores se podría hablar de élites, o de líderes, o jefes. 
vistas en las mismas. "En los países en que no existe división de poderes, como es en la mayoría de los regímenes militares, o en aquellos en que existe una división artificial (por ejemplo, Méjico, Irán) los directores se encuentran en las camarillas, en comités sanitarios y en puestos de mando en la burocracia pública (Ministerio de Sanidad, división de sanidad de planificación nacional, etc.). A lo largo del continuo división no-división de poderes pueden colocarse los sistemas políticos de acuerdo al grado de división de poderes, y la búsqueda de los directores presupone un entendimiento del sistema político en cada país. ${ }^{12}$ Hay naciones en donde los médicos personales de los jefes supremos son los directores más importantes aunque no ocupen ningún cargo público o administrativo; en otros los jerarcas de planificación nacional o los directores de hospitales universitarios 0 incluso asesores extranjeros se encuentran entre los directores más importantes. La identificación de los directores de sanidad es un ejercicio complejo, como los estudiantes de estructuras de poderes pueden atestar, y las técnicas empleadas en la identificación de decisores en los estudios de comunidades pueden ser de utilidad. ${ }^{13}$ No hay que olvidar que, dada la amplitud del término salud, diferentes aspectos de la política sanitaria son definidos por diferentes directores o élites; por ejemplo, los aspectos de nutrición se deciden en el Ministerio de Agricultura, y los de sanidad en el de Obras Públicas. Sin embargo es necesario saber quiénes son los directores y cuáles son sus criterios para las decisiones, si es que queremos llegar a comprender todo el proceso decisorio y al final el fallo en la implementación. En particular hace falta conocer de los directores sus ideologías políticas, su experiencia profesional (un sanitario tomará decisiones diferentes a un médico cirujano), la clase social, las relaciones con grupos de presión (asociaciones de médicos, grupos farmaceúticos). Es necesario saber también qué elemen-

11. En algunas circunstancias en los Estados Unidos el presidente puede vetar un proyecto de ley sin hacer nada, es decir, a través del llamado «veto del boisillo». En otros países con separación de poderes el presidente puede imponer su preferencia para los gastos de inversión pero no para los de operación. Es decir, que cada sistema político tiene su manera de delimitar los poderes constitucionales de las diferentes ramas de gobierno. Una colección interesante de artículos sobre las funciones y poderes de los congresos y parlamentos en varios países del mundo es Allais Kornberg y Lloyd D. Musolf, eds., Legislatures in Developmental Perspective (Durham, N. C.: Duke University Press, 1970).

12. Naturalmente que la distinción división y no división de poderes no es un intento de tipología de sistemas políticos sino una clasificación que para nuestro es. tudio resulta más conveniente que las de Lipset, Apter y otros.

13. La bibliografía sobre el tema es extensísima sin que se haya llegado a es. tablecer en definitiva qué método es el más apropiado para cada circunstancia. 
tos usan en sus decisiones como los tipos de estadísticas (si es que se usan), naturaleza de las asesorías, etc. Las formas en que se toman las decisiones son igualmente importantes, si son decisiones individuales 0 si por el contrario son decisiones colectivas, qué tipo de discusión lleva a la definición de políticas y que grupos se consultan o se escuchan (gobernadores, grupos cívicos, directores de hospitales).

El siguiente es un ejemplo de estilo decisorio. En junio de 1972, durante la visita de la primera dama de Irán a una de las provincias, al pasar por uno de los barrios pobres de la capital quedó impresionada por la situación de hambre de los niños. Inmediatamente ordenó al primer ministro que le acompañaba que mandara preparar un programa de nutrición para la ciudad. La prensa presentaba el incidente resaltando el espíritu de benevolencia y la determinación de la emperatriz por resolver los problemas sociales de la nación elogiando su estilo de «decisiones sobre la marcha». Pocos días después llegaba a la ciudad un equipo de «expertos» para preparar el programa de nutrición que consistía en distribución de alimentos. Unas semanas después visitamos las oficinas del presupuesto en planificación nacional en donde explicaron que aproximadamente medio millón de dólares habían sido rápidamente asignados para el programa de nutrición. Al mismo tiempo, en el Ministerio de Sanidad nos informaron que en el Ministerio no había nutricionistas. Como puede comprenderse, decisiones sobre la marcha permiten muy poco tiempo a los programadores para traducir las políticas en programas, estudiar alternativas y prever obstáculos a su implementación. Las posibilidades de que se implementen disminuyen e incluso cuando se implementan puede ser que resuelvan solamente los efectos del problema pero no la causa, como es el caso en el ejemplo. Un programa de nutrición que consiste solamente en distribución de alimentos no soluciona la causa de la malnutrición que entre otras son la pobreza, la ignorancia sobre el valor nutritivo de los alimentos, los hábitos, etc. Queda claro que el estilo decisorio y las relaciones entre los decisores de políticas y los programadores determinan en algún grado la probabilidad de implementación de un programa.

Llamamos programadores e implementadores a las personas que respectivamente traducen las decisiones iniciales o políticas sanitarias en programas y a las que los implementan. La mayoría de los actores en este grupo se localizan en la administración pública pero también se pueden localizar en el sector privado (juntas directivas de hospitales, facultades de medicina privadas, agencias voluntarias) y en las agencias internacionales (OMS, OPS, UNICEF). Los programadores deciden los programas que se van a llevar a cabo, por ejemplo, construcción de acue- 
ductos, control de enfermedades venéreas o educación sanitaria, y también el número de actividades y su modalidad, veinte acueductos en zonas rurales con plantas de purificación, distribución de dos millones de filtros domésticos de agua. Encontramos que hay un gran número de decisiones a diferentes niveles de la administración pública; algunas decisiones son generales y otras muy específicas, algunas se toman a nivel de comité técnico en el Ministerio o en la junta directiva de una agencia autónoma o descentralizada, y otras decisiones las hacen individuos profesionales como los jefes de división o de unidades y sus subalternos, y otras decisiones las hacen personal subprofesional o no profesional como pudiera ser la decisión de actualizar un cardex de personal o inventarios en los almacenes de drogas, o la modalidad de las visitas de inspectores sanitarios.

Los implementadores son personas diferentes de los programadores: estos programas que los médicos del Estado deben hacer seis consultas por hora y los médicos (que son los implementadores) deciden informalmente hacer cinco o hacer las seis en media hora y utilizar el resto del tiempo en consultas de pacientes privados. Los programadores determinan el número de vacunas que se deben poner en una campaña masiva y todos los detalles de acuerdo a normas de programación, y los vacunadores (implementadores) deciden poner las vacunas o tirar algunas miles al río. Naturalmente los sistemas de supervisión existen para reducir a un mínimo las disparidades entre los programas y la implementación. Sin embargo sucede: (1) Los programadores en ciertas ocasiones carecen de poder jurídico, político e incluso administrativo sobre los implementadores. Éste es el caso, por ejemplo, cuando las acciones del Ministerio o de una agencia de sanidad las implementan personal de otro Ministerio (Agricultura para veterinaria o nutrición, Obras Públicas para ingeniera sanitaria). O cuando los implementadores pertenecen a unidades político-administrativas autónomas de hecho o de derecho, como es el caso de ciudades cuya importancia política y económica sobrepasa al de la provincia en donde están localizadas; en este caso las autoridades provinciales de sanidad carecen de capacidad política de supervisión, una situación que se presenta con frecuencia en los esfuerzos de regionalización de servicios de atención médica o en el uso de servicios de emergencia. A veces los hospitales universitarios son más poderososs que las secretarías provinciales de sanidad. (2) La supetvisión en muchos sistemas de sanidad es deficiente y es difícil evitar lo que en administración pública se llama la «fuga de autoridad»; cada vez que se transmite el programa a nivel de implementación más específico hay una nueva posibilidad de modificar la decisión inicial con una interpretación dentro 
de unos márgenes cuya amplitud depende de lo que pudiéramos llamar el estilo administrativo del servicio civil o burocracia. (3) Los supervisores también toman decisiones pudiendo estar más interesados en supervisar unos programas más que otros, o ciertos aspectos de un programa. Por ejemplo, todavía no se ha estudiado en ningún país la relación entre la policía y la administración de sanidad, aunque en muchos países la policía es la fuerza pública con poder de poner sanciones contra las violaciones del código sanitario. (4) Puede también suceder que los programas fueron mal diseñados o son inadecuados para ciertos ambientes y por tanto resulta imposible su implementación; los implementadores necesitan hacer cambios sobre la marcha o en el campo con repercusiones en otros programas de sanidad.

De todo lo dicho se desprende que el estudio del grupo de decisores de programación e implementación es sumamente complejo, puesto que incluye un gran número de personas desde los secretarios y directores generales de las instituciones de sanidad y aquellas otras instituciones que están indirectamente relacionadas con la salud hasta el personal auxiliar de los mismos. Los expertos en administración pública han investigado muchos aspectos de la burocracia pero raramente se ha estudiado el proceso decisorio en sí mismo y menos veces aún se ha estudiado el del sector sanitario. ${ }^{14}$

Una vez que se han identificado los decisores y los niveles a que se toman las decisiones (jefe de división, personal auxiliar, etc.), es necesario estudiar, como en el caso de los directores, los criterios y motivación de las decisiones. La literatura sociológica ha establecido posibles factores de influencia o posibles variables independientes tales como valores culturales y étnicos, clases sociales, ideologías, experiencia profesional, edad, sexo y religión. Mucha de esta información se puede recoger a través de encuesta pero hay otro tipo de información que resulta más difícil a no ser que se utilice el método de participación y observación. Nos estamos refiriendo a las decisiones que resultan de las luchas internas entre grupos dentro de una misma agencia o entre grupos de vatias agencias, por ejemplo, entre los médicos del Ministerio y los médicos del seguro, o entre los arquitectos y los médicos del Ministerio, o entre los médicos y los administradores de hospitales, entre grupos políticos diversos o entre rivalidades que emergen alrededor de personali-

14. Entre los pocos estudios sobre el sistema decisorio se destaca el excelente trabajo de Anthony Downs, Inside Bureaucracy (Boston: Little Brown, 1967), y el estudio ya clásico de Herbert Simon, Administrative Bebavior. A Study of Decision Making Process in Administrative Organization (Nueva York: Free Press, 1945) ninguno de los dos sobre el sector salud. 
dades. En otras palabras, para llegar a comprender el sistema decisorio del sector sanitario es necesario conocer la estructura informal de sus organizaciones.

El tercer grupo decisorio es el de los consumidores de salud, que son los ciudadanos. En la teoría moderna de administración de empresas se ha empezado a incluir a los consumidores como parte de la empresa junto a los accionistas, ejecutivos y los trabajadores. Se piensa que si la moral de los trabajadores influye en el rendimiento de la empresa, la disposición del consumidor es también importante, puesto que es él quien decide utilizar el producto y la forma de utilizarlo, y por tanto puede decidir en definitiva si la empresa sigue funcionando o no. Pensamos que esta lógica conviene llevarla a la administración pública, dada la separación mayor cada día entre los administradores del Estado y los ciudadanos, incluso en aquellas sociedades en donde los administradores públicos deben responder por sus acciones a representantes elegidos por la ciudadanía. El ciudadano como consumidor de servicios públicos de salud puede decidir usarlos o no usarlos, y decidir también la forma en que los va a utilizar. Por ejemplo, programas de nutrición han tenido menos éxito del esperado porque los consumidores no han aceptado las innovaciones alimenticias. En Centroamérica, una gran parte de la población ha rechazado las tortillas de maíz fortificadas por extrañar el sabor. Actualmente, el INCAP (Instituto de Nutrición para Centro América y Panamá) de las Naciones Unidas está investigando la tolerancia o aceptación del sabor, color y consistencia de tortillas con diferentes aditivos nutricionales. Esta actitud de tener en cuenta la opinión del consumidor de un servicio de salud corresponde a los estudios de mercado tan corrientes en el sector privado empresarial. Un ejemplo clásico, por ser tan corriente en la mayoría de los países del mundo en vías de desarrollo, es la subutilización de hospitales en las zonas rurales. A pesar de que los indicadores de salud manifiestan una situación precaria en esas zonas, por razones múltiples y complejas la tasa de ocupación de camas por mes es en los hospitales rurales muy baja; los campesinos deciden no utilizarlos o irse a los hospitales urbanos. Como resultado de las decisiones de los consumidores, los programas de regionalización de servicios planeados de acuerdo a técnicas modernas de distribución espacial han fracasado. En un estudio en el valle del Cauca (Colombia) se vio que muchas campesinas prefieren ser atendidas por parteras tradicionales en vez de ir al hospital. Trabajo de campo detallado demostró que la atención dada por las parteras tradicionales era desde algunos puntos de vista de las consumidoras de servicios más adecuada que la que se daba en los hospitales, había más entendimiento, accesibilidad e 
interés por parte de las parteras sobre el bienestar de la parturienta que por parte del médico; en algunos casos, la falta de recursos en los hospitales condujo a dar una atención médica deficiente con largas horas de espera que en una situación extrema resultó en un parto sin asistencia en la misma sala de espera.

En muchos países del mundo se está empezando a hacer esfuerzos por organizar las comunidades sobre todo en las áreas rurales con el fin de facilitar la articulación de las demandas y el conocimiento de las necesidades de la población y poder incorporar estas dimensiones en la planificación sanitaria. ${ }^{15}$ En Malasia, por ejemplo, se han formado los comités de desarrollo en los kampongs; en Colombia las asociaciones de usuarios campesinos; en Méjico las asociaciones de colonias proletarias y los ejidos (que datan de la época de la revolución). En relación al sector sanitario, uno de los países en donde se ha entendido bien la necesidad de organizar la comunidad ha sido Panamá. El Ministerio de Sanidad Pública ha organizado comités de sanidad en los pueblos a los que pertenecen todos los adultos de la comunidad. La junta directiva elegida por el comité transmite de una parte las necesidades de la comunidad al Ministerio y de otra las instrucciones y directivas del Ministerio a la comunidad. Los comités de sanidad tienen diferentes proyectos relacionados con la comunidad; por ejemplo, proyectos relacionados con la comunidad, proyectos de nutrición con huertos comunitarios en donde se cultivan algunos de los alimentos necesarios para una dieta balanceada. Los comités también facilitan las actividades de los centros de salud proveyendo transporte, alojamiento a personal paramédico, a los vacunadores, etc.

Éste no es el lugar para hacer un análisis crítico de los programas de organización de la comunidad y sus efectos en planificación e implementación de sanidad. La impresión que tenemos es que los esfuerzos de organizar la comunidad no están dando los resultados esperados que faciliten la programación e implementación porque no se están utilizando para conocer las necesidades de la población y comprender el sistema decisorio de los consumidores, al contrario se utilizan para imponer los programas de gobierno, en otras palabras, tienden a convertirse en instrumentos de control. La comunicación de doble dirección (que constituye otro punto de comunicación o linkage) que los programas de acción comunal deben realizar se convierte en una sola dirección que es la del programador o implementador al consumidor. Así, el mismo pro-

15. La mayoría de los esfuerzos se han hecho en el sector agrícola, sobre todo en la organización de cooperativas, y en los tugurios de las grandes ciudades a través de organizaciones de acción comunal. 
grama de Panamá tan bien diseñado y concebido ha tenido sus limitaciones. Por ejemplo, en el caso de los huertos comunales algunas de las hortalizas que los nutricionistas consideraron importantes y que fueron cultivadas por la comunidad no llegaron a consumirse ni a comercializarse y se echaron a perder porque los habitantes las desconocían y no las incorporaron a sus dietas. En otras ocasiones las organizaciones comunales han rechazado los controles de la burocracia creándose situaciones de conflicto. En Malasia, por ejemplo, las cooperativas de campesinos se organizaron tan bien que se convirtieron en un grupo de presion y empezaron a tomar decisiones que con frecuencia se oponían a las decisiones de los programadores y, en 1974, el gobierno disolvió las cooperativas agrícolas. En Colombia las farmacias comunales fracasaron sin que nunca se llegara a analizar sus causas que conllevan a altas pérdidas monetarias. Con frecuencia hemos oído las críticas de los médicos y de otros trabajadores en sanidad pública que consideran las demandas de la población organizada como una interferencia en sus intentos de planificación sanitaria. En definitiva, a pesar de los muchos intentos, no se ha conseguido incorporar a los consumidores de una manera útil a la planificación sanitaria.

Conviene insistir que el conocimiento de las actitudes de la población sobre los programas y servicios de sanidad debe ser una parte integrante de la planificación sanitaria, y hay que añadir que esta necesidad es más urgente en aquellas sociedades en donde los consumidores tienen canales limitados de expresión por falta de medios de comunicación de masas, de partidos u organizaciones políticas independientes como es corriente en casi todos los sistemas autoritarios y algunos regímenes militares.

Igualmente la necesidad de tener en cuenta el proceso decisorio de los consumidores se hace imperativo en aquellas sociedades con sistemas de valores múltiples bien sea por la presencia de grupos étnicos o por la presencia de clases sociales distanciadas. Será bien difícil para un profesional, ya sea médico, economista, ingeniero sanitario, nutricionista, etc., comprender las reacciones de campesinos analfabetos y sus razones de rechazo de servicios sanitarios a no ser que haga el esfuerzo de comprender su proceso decisorio. En situaciones así, se hace imperativa la utilización de antropólogos sociales.

$\mathrm{El}$ cuarto grupo lo constituyen los grupos de presión o de intereses especiales, y es quizás el más conocido en sociología a través de los muchos trabajos que se han hecho sobre los mismos aunque en el sector sanitario los estudios son pocos y casi todos se han realizado en los 
países industriales. ${ }^{16}$ Las asociaciones de consumidores funcionan a veces como grupos de presión, éste es por ejemplo el caso del Sierra Club en los Estados Unidos, muchas de cuyas actividades están dirigidas a luchar contra la contaminación ambiental. Pero hay otros muchos grupos de presión que representan los intereses de proveedores privados de componentes de salud como son las asociaciones profesionales de médicos, enfermeras, de sanitarios o las industrias que producen bienes para la salud como las firmas farmacéuticas, o las asociaciones que prestan servicios sanitarios como son las asociaciones hospitalarias, los grupos de seguros médicos; hay otras asociaciones que representan intereses especiales con repercusiones en programas sanitarios como son grupos religiosos, sindicatos obreros adscritos a la seguridad social, el Consejo Mundial de Población, etc. Algunos grupos de intereses funcionan a través de los grandes carteles internacionales como son en particular las firmas farmacéuticas con sede en Alemania, Suiza o los Estados Unidos, o a través de agencias internacionales de desarrollo. ${ }^{17}$

A pesar de que el estudio de los grupos de presión está bien establecido en las ciencias sociales, hay muy pocas investigaciones sobre la manera en la que influyen en el proceso decisorio en las fases de programación y de implementación. Conviene insistir que los estudios que existen han examinado los grupos de presión desde el punto de vista de las influencias ejercidas en lo que hemos llamado decisiones iniciales o políticas sanitarias. Pero los grupos de presión participan también en la fase de programación y de implementación no solamente a través de presiones, sino de acciones. Por ejemplo, la industria farmacéutica internacional ha desequilibrado los intentos de distribución de productos farmacéuticos de algunos gobiernos y la producción de genéricos vendiendo a precio más bajo de costo de producción. Los gtandes consorcios internacionales pueden absorber pérdidas en un país durante períodos prolongados y así desacreditan la capacidad de las agencias de gobierno de proveer medicinas a precio reducido; el gobierno bajo críticas y presiones desmonta sus agencias y el sector privado consigue de nuevo monopolizar el mercado.

16. Véase, por ejemplo, Barbara y John Ehrenteich, The American Health Empire: Power, Profits, and Politics (Nueva York: Random House, 1970); Bernard R. Blishen, Doctors and Doctrines. The Ideology of Medical Care in Canada (Toronto: University of Toronto Press, 1969); Eugene Feignold, Medicare: Policy and Politics (San Francisco: Chandler, 1966).

17. Quizás el único estudio que existe sobre el tema es Carlos Bertero, Drugs and Dependency in Brazil: An Empirical Study of Dependency Tbeory. The Case of the Pbarmaceutical Industry (Ithaca, Nueva York: Cornell Latin American Dissertation Series, 1972). 
La identificación de los grupos de presión en el sector privado puede ser una tarea laboriosa en casi todas las sociedades pero se convierte muy compleja en las industrializadas por el gran número de asociaciones y organizaciones que directa o indirectamente están relacionadas con la salud. Si por ejemplo consideramos la contaminación del ambiente o la seguridad ocupacional como aspectos de la salud, entonces es necesario estudiar las políticas y las actividades de las industrias respecto a ellas. Una vez identificados los grupos de influencia hay que estudiar el proceso decisorio, lo que resulta muy difícil por las pocas facilidades que estas organizaciones dan a las investigaciones que no son financiadas por ellas mismas. Las ilegalidades y la corrupción en sus relaciones con los gobiernos son frecuentes $y$, como se puede esperar, no tienen interés en que se expongan estas irregularidades. Los estudios de un caso concreto a través de documentos y conversaciones con ex ejecutivos es la técnica más apropiada para investigar la mayoría de los grupos de interés; los grupos profesionales o de trabajadores y de consumidores se pueden estudiar a través de encuestas y quizá de observaciones directas.

\section{Coordinación en la programación y en la ejecución}

Hay muy pocos estudios sobre los aspectos de coordinación entre agencias de gobierno así como también entre los sectores público y privado. Sin embargo, cuando la Agencia $A$ está encargada de recursos humanos para la salud y la agencia $B$ de la atención médica en zonas rurales, es necesario que las decisiones de las dos agencias estén sincronizadas o de lo contrario se acabará con un exceso de ciertas clases de personal en las ciudades y con escasez de otras en las zonas rurales.

En muchos países los administradores de sanidad se dan cuenta de la falta de coordinación que existe entre las agencias pero falta un marco conceptual que sirva para recoger las experiencias y ayude a buscar soluciones. Uno de nuestros entrevistados comentaba: «El Ministerio es responsable por el funcionamiento de las oficinas provinciales de sanidad pero no tenemos ningún control, y no es posible supervisarles porque los directores provinciales de Sanidad hacen más o menos lo que les da la gana (su nombramiento lo hace el emperador). Las normas que les mandamos las cambian de acuerdo a su propio criterio. Éste es el problema. Cuando decimos que se debe dar prioridad a la campaña contra el cólera, en las provincias pueden decidir otra cosa.»

Pensamos que es necesario distinguir entre coordinación vertical y coordinación horizontal e indicar que los dos tipos se dan dentro de 
TABLA 1

Representación esquemática de la coordinación decisoria en una burocracia pública

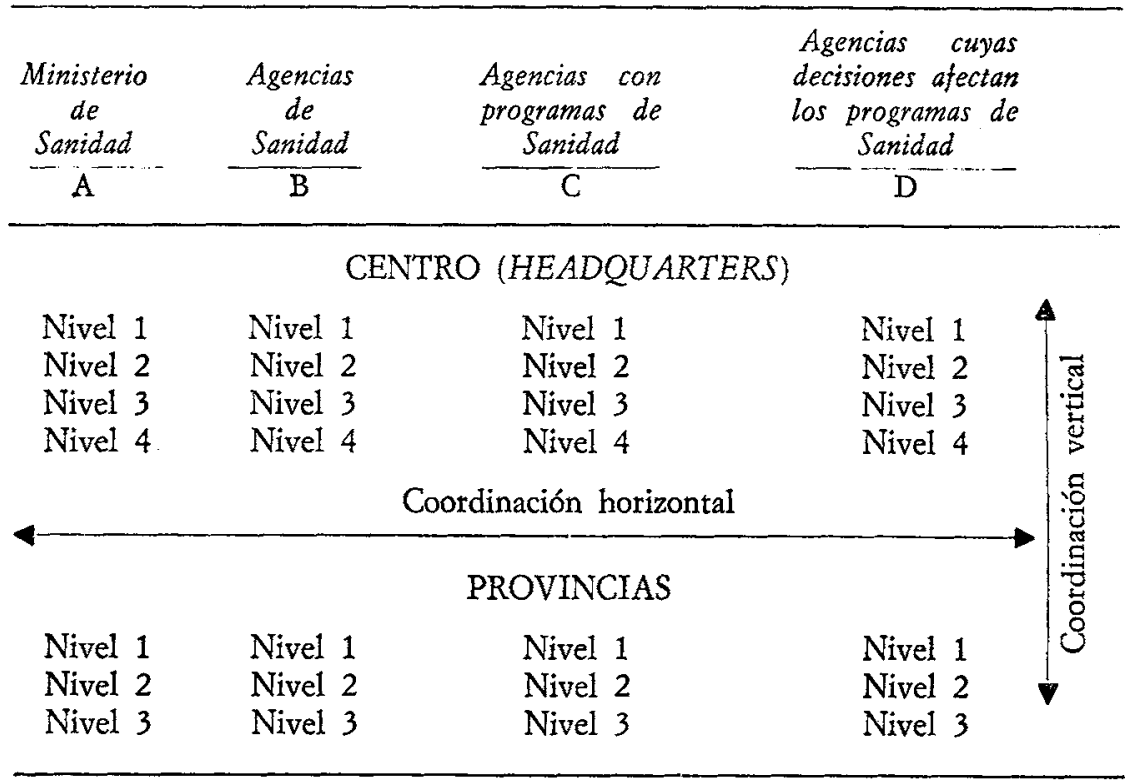

Nota: Definición de sanidades por niveles:

Centro:

Nivel 1: Ministros, directores generales, presidentes, y presidentes de junta

Nivel 2: Sub-secretarios, sub-directores

Nivel 3: Jefes de departamentos

Nivel 4: Jefes de unidades o secciones

Provincias:

Nivel 1: Directores generales, delegados

Nivel 2: Sub-secretarios, jefes de departamentos

Nivel 3: Jefes de unidades o secciones

agencias y entre agencias. La Tabla 1 es una representación esquemática de los dos tipos de coordinación. Coordinación horizontal es la que existe entre unidades de una agencia o agencias que están al mismo nivel decisorio, tanto a nivel central como provincial y local (o cuantos niveles verticales hubiera). Los problemas y las saluciones para la coordinación en el nivel central (Ministerios, seguros sociales) son diferentes de los de la periferia (provincias, delegaciones) pero la modalidad de los problemas y sus soluciones son una función de la modalidad de los problemas en el nivel central; en otras palabras, los problemas de coordinación son diferentes en el centro y en la periferia pero están relacionados. 
TABLA 2

Grado, institucionalización y calidad de la coordinación en el sector sanidad de Irán

\begin{tabular}{|c|c|c|c|}
\hline Unidades & Grado & $\begin{array}{l}\text { Instituciona- } \\
\text { lización }\end{array}$ & Calidad \\
\hline
\end{tabular}

CENTRO

HoRIzONTAL

Dentro de A

Nivel 1

Nivel 2

Nivel 3

Nivel 4

Entre A-B

Nivel 1

Nivel 2

Nivel 3

Entre A-C y A-D

Nivel 1

Nivel 2 y $3 \& 4$ falta de coordinación

bien a regular

malo

falta de coordinación

$\begin{array}{cl}\text { si } & \text { ni } \\ \text { si/no } & \text { mala } \\ \text { no } & \text { falta de coordinación }\end{array}$

na

bien a regular

regular a malo

falta de coordinación

$\begin{array}{cl}\text { na } & \text { na } \\ \text { si } & \text { regular } \\ \text { si/no } & \text { mala } \\ \text { no } & \text { falta de coordinación }\end{array}$

si ni

no falta de coordinación

VERTICAL

Dentro de A malo

Dentro de B

yC \& D ni ni ni

\section{PROVINCIAS}

HoRizONTAL

Dentro de A

Nivel 1

na

si/no

mala

Nivel 2 \& 3 ni

na na

ni ni

Entre $A-B$ y $A-C$ y A-D malo ni mala

Nota: La Tabla 2 debe ser relacionada con la Tabla 1 al leerse: $\mathrm{ni}=$ no información

Grados están definidos como cantidad de interacción. $\mathrm{na}=$ no aplicable

Grados y calidad están categorizados en la siguiente forma: excelente, bueno, regular, malo, y falta de coordinación.

Institucionalización está dividida en sí o no. La categoría si/no significa que algunas relaciones están institucionalizadas y otras no. 
La coordinación vertical es la que se da (o se debiera dar) entre unidades que están a diferentes niveles decisorios, por ejemplo entre la división materno-infantil del Ministerio y la división materno-infantil del Estado (provincia o departamento). El tipo de relaciones entre los diferentes niveles varía de acuerdo a la constitución del país; en algunos los niveles verticales son independientes, como sucede en los países federales; en otros los niveles provinciales sólo tienen poder decisorio en la fase de implementación.

Es importante empezar a cuantificar la coordinación para poder hacer estudios comparativos entre países y aprender cómo la falta de coordinación en las decisiones afecta la implementación de programas. La Tabla 2 presenta un intento de operacionalización con la información recibida en el estudio del sector sanitario de Irán. Como se puede observar, sólo se han operacionalizado tres posibles variables (más estudios empíricos ayudarán a encontrar más o quizás otras más pertinentes). El grado de coordinación mide el número de contactos entre unidades y es cuantificable. La variable calidad mide la efectividad de los contactos y se podría cuantificar a través de encuestas por medio de la escala Lijert. La variable institucionalización mide la naturaleza de la coordinación y ha sido dicotomada: si se hace de acuerdo a normas establecidas existe institucionalización y si se hace informalmente no existe. Se puede pensar que si los contactos son reducidos y poco efectivos y no hay institucionalización la coordinación será baja.

En los trabajos que hemos llevado a cabo en varios países se ha observado que la falta de coordinación no solamente lleva a la duplicación de servicios y a la pérdida de los recursos escasos sino que también es una causa frecuente del fallo en la implementación (recuérdese que las agencias y unidades que programan son a veces diferentes de las que implementan). Un administrador en Irán hacía una astuta observación cuando comentaba: «La recolección de estadísticas se deteriora más y más a medida que aumenta el número de agencias que prestan servicios de salud,» $\mathrm{Y}$ añadía: «Originalmente el Ministerio de Desarrollo Rural y de Cooperativas se creó para resolver todos los aspectos de desarrollo de las zonas rurales. Pero esto no es posible en relación a los problemas de salud. No se puede tener un dispensario de salud del Ministerio de Desarrollo rural aquí y dos kilómetros más allá en el pueblo uno del Ministerio de Sanidad, especialmente cuando hay que enfrentarse con enfermedades transmisibles.»

Hasta el momento tres son las soluciones que con frecuencia se han sugerido para aumentar la coordinación de decisiones: (1) la disminución del número de agencias que trabajan en sanidad; (2) la creación de un 
superministerio que tenga poder decisorio sobre los demás y supervise la coordinación; y (3) la organización de un consejo interministerial para la sanidad. Está en boga hoy día la corriente de integración de servicios ya sea entre las agencias o entre divisiones político-administrativas. En Panamá, por ejemplo, se ha empezado la integración de los servicios de salud del Ministerio y los del seguro social en la provincia de Colón. En Colombia se han integrado recientemente los servicios de la ciudad de Cal con los del departamento de Valle, y la mayoría de los hospitales de la Iglesia han sido también integrados con los del servicio de salud pública. En Irán se estaba preparando en 1972 un proyecto de ley para integrar los diferentes sistemas de seguridad social en uno sólo. Es cierto que disminuyendo el número de agencias se disminuye una parte del problema de coordinación horizontal entre las agencias pero se aumenta dentro de las agencias que las incorpora (aunque hay que reconocer que dentro de una agencia la coordinación es más sencilla). Sin embargo, a medida que aumenta la complejidad de la vida moderna la burocracia tiende a crecer también, $\mathrm{y}$ no se sabe qué es más deseable: unas pocas agencias de proporciones gigantescas o un mayor número de instituciones más pequeñas. Los peligros de concentrar el poder en manos de unos pocos burócratas no se deben subestimar. La segunda solución es también muy común; por ejemplo, en Malasia se organizó en los primeros años de la década de los sesenta el Ministerio de Desarrollo Rural que llegó a tener poder sobre todos los demás Ministerios por el hecho de que su ministro era al mismo tiempo vicepremier. Ness en su libro ha descrito muy bien los resentimientos que se crearon entre los Ministerios como resultado de esa situación y cómo terminó el experimento. ${ }^{18}$ En casi todos los países en vías de desarrollo las agencias de planificación nacional han asumido un poder extraordinario sobre todo en los programas de inversión convirtiéndose de hecho en superministerios $\mathrm{y}$, sin embargo, no han podido resolver la falta de coordinación. La tercera solución es igualmente frecuente en muchos países, y nuestra experiencia en Irán y Colombia sugiere que los consejos nacionales de sanidad no han funcionado muy efectivamente. En parte, el fracaso de los consejos se debe a que no tienen poder coercitivo sobre las agencias sanitarias, esto supondría que estarían por encima de los ministros. En definitiva no se ha encontrado todavía ningún sistema administrativo que facilite el sistema de coordinación. ${ }^{19}$

18. Gayl Ness, Bureaucracy and Rural Development in Malaysia (Berkeley: University of California Press, 1967).

19. En otro trabajo todavía inédito hemos discutido con más detalle las razones por las que parecen haber fracasado los consejos nacionales de salud. Véase 
Los fallos en la implementación de los programas afectan el proceso decisorio en sí mismo. Cuando el decisor prevé que por falta de coordinación o por otra causa sus decisiones no se van a implementar o van a ser modificadas, la pregunta lógica que se hace es: ¿qué más da hacer buenas o malas decisiones? Uno de nuestros entrevistados se expresó casi con estas mismas palabras. Los expertos sobre desarrollo han usado el modelo del círculo vicioso acumulativo para explicar la causalidad circular del subdesarrollo. ${ }^{20}$ Se podría aplicar el modelo para explicar el proceso de toma de decisiones en muchas administraciones: el frecuente fallo de implementación de decisiones produce un interés limitado en buscar información, en la supervisión y en la investigación que a su vez lleva a decisiones incorrectas que a su vez producen fallos en la implementación. Si se entiende el fallo de implementación de esa manera queda claro que la única manera de romper el círculo vicioso es atacándole en todas partes al mismo tiempo. Frecuentemente se oye, por ejemplo, que la planificación sanitaria es de poca utilidad porque los datos de que se disponen son escasos y de poca exactitud; puede que esto sea así, pero el modelo nos indica que, por muy buenas que sean las estadísticas de sanidad, no por ello mejorarán los servicios de sanidad si no se mejoran otros aspectos del proceso decisorio.

\section{Antonio Ugalde}

Department of Sociology

University of Texas

Austin, Texas 78712

USA

«Health Decision Making in Developing Nations: A Comparative Analysis», 1974 (mimeo).

20. Gunnar Myrdal, Economic Theory and Underdeveloped Regions (Nueva York: Harper and Row, 1957). 